\title{
Pancreatitis aguda durante el embarazo secundaria a dislipidemia mixta. Reporte de un caso
}

\author{
Félix Fernando León Carrillo, ${ }^{*}$ María Guadalupe Hernández Landeros ${ }^{\ddagger}$
}

\section{RESUMEN}

Femenino de 16 años que cursaba con un embarazo de 17 semanas de gestación al momento de su ingreso al hospital; refería dolor intenso en el epigastrio posterior a transgresión alimentaria, acompañado de náusea, que llegaba a la emesis de contenido gastroalimentario; era portadora de diabetes mellitus tipo 1 , además de antecedentes de pancreatitis aguda grave secundaria a hiperlipidemia a los 14 años, apendicetomía a los 15 años e infección de vías urinarias en el primer trimestre; se realizaron paraclínicos con resultados relevantes, a razón de leucocitos 15,600, amilasa 1,117 U/L, lipasa 1,200 U/L, suero lipémico ++++, triglicéridos 2,840, colesterol 705, HDL $20 \mathrm{U} / \mathrm{L}$, DHL $289 \mathrm{U} / \mathrm{L}$, TGO 14 U/L, TGP 6 U/L, FA $60 \mathrm{U} / \mathrm{L}$ y bilirrubina total $0.5 \mathrm{pg} / \mathrm{dL}$, con lo cual se llegó al diagnóstico de pancreatitis aguda secundaria a dislipidemia mixta. Dado que se realizaron estudios de laboratorio en los hermanos y la rama femenil fue positiva, con elevación de colesterol y triglicéridos, concluimos que se trataba de una dislipidemia familiar combinada, lo que motivó su publicación.

Palabras clave: Embarazo, pancreatitis, dislipidemia familiar.

\section{SUMMARY}

Sixteen-year-old female, with a 17-week pregnancy at the time of admission to the hospital; she referred epigastric pain after food transgression, accompanied by nausea, which reached emesis with gastro-alimentary content; she had diabetes mellitus 1 plus a history of severe acute pancreatitis secondary to hyperlipidemia at age 14, appendectomy at age 15 and a urinary tract infection in the first trimester; paraclinical tests were performed, with the following relevant results: leukocytes 15,600 , amylase 1,117, lipase 1,200, lipemic serum ++++, triglycerides 2,840, cholesterol 705, HDL 20, DHL 289, SGOT 14, SGPT 6, FA 60 and total bilirubin $0.5 \mathrm{pg} / \mathrm{dL}$, which led to the diagnosis of acute pancreatitis secondary to mixed dyslipidemia. Laboratory tests were performed in her siblings and the female branch was positive, with elevation of cholesterol and triglycerides; therefore we concluded that it was a familial combined dyslipidemia, which motivated its publication.

Key words: Pregnancy, pancreatitis, dyslipidemia.

\section{RESUMO}

Feminino de 16 anos com 17 SDG no momento da internação, referindo dor epigástrica após transgressão alimentar acompanhada de náusea e emese de conteúdo gastroalimentario, portadora de DM tipo 3 e antecedente de pancreatite aguda grave secundária a hiperlipidemia aos 14 anos; além de história de pancreatite aguda grave secundária a hiperlipidemia aos 14 anos, apendicectomia aos 15 anos e infecção do trato urinário no primeiro trimestre; foram realizados estudos pré-clínicos com resultados relevantes na proporção de leucócitos 15.600, amilase 1117, lipase 1200, soro lipêmico ++++, triglicerídeos 2840, colesterol 705, HDL 20, DHL 289, TGO 14, TGP 6, FA 60 e bilirrubina total $0.5 \mathrm{pg} / \mathrm{dL}$, o que levou ao diagnóstico de pancreatite aguda secundária a dislipidemia mista e o estudo laboratorial realizado em irmãos permanece positivo na rama feminina da família. Concluímos que é uma dislipidemia familiar combinada, motivo de sua publicação.

Palavras-chave: Gravidez, pancreatite, dislipidemia.

\section{INTRODUCCIÓN}

Se trató de paciente femenino de 16 años con un embarazo de 17 semanas de gestación (SDG) que acudió al hospital a la presentación de dolor abdominal epigástrico intenso secundario a transgresión dietética (ingesta de colecistoquinéticos), acompañado de vó-

\footnotetext{
* Hospital General de Pachuca, Hidalgo.

‡ Hospital Regional de Aguascalientes, Ags.

Unidad de Cuidados Intensivos del Hospital Regional, ISSSTE, León, Gto.
}

Recepción: 23/01/2018. Aceptación: 11/05/2018.

Este artículo puede ser consultado en versión completa en http://www.medigraphic.com/medicinacritica mito y mal estado general. Tenía antecedentes de un cuadro de pancreatitis dos años antes secundario a dislipidemia, diabetes mellitus en tratamiento con insulina 15 y $10 \mathrm{U}$, y apendicectomía un año atrás. Se realizaron paraclínicos, con los siguientes hallazgos: suero lipémico ++++/++++. Se ingresó a la unidad de terapia intensiva; se inició el manejo para pancreatitis aguda secundaria a dislipidemia, así como embarazo de 17 SDG por FUM. La pancreatitis es un evento raro durante el embarazo: 1: 1,000 o 1:10,000. ${ }^{1} \mathrm{La}$ etiología más común es de origen biliar; ${ }^{2,3}$ asociada con dislipidemia es aún más rara, siendo una complicación grave y potencialmente fatal.

\section{CASO CLÍNICO}

Paciente primigesta de 16 años, con los siguientes antecedentes: pancreatitis aguda grave secundaria a hiperlipidemia a los 14 años, con estancia hospitalaria de dos meses, con secuela de diabetes mellitus tipo 1; dislipidemia con manejo de bezafibrato y pravastatina, con mal apego al tratamiento; quirúrgicos positivos a razón de apendicetomía a los 15 años; resto de los antecedentes, interrogados y negados. Antecedentes ginecoobstétricos: menarca a los 12 años, eumenorreica, inicio de la actividad sexual a los 16 años con una sola pareja; no uso de método de planeación familiar ni detección de cáncer de mama o cervicovaginal; gestas: 01 , abortos: 00, partos: 00 y cesáreas: 00. Embarazo actual de 17 semanas de gestación por fecha de última menstruación, infección de vías urinarias en el primer trimestre con tratamiento médico no especificado; control prenatal en cuatro ocasiones.

Inició su padecimiento actual el 7 de enero de 2012 posterior a una transgresión alimentaria (ingesta de alimentos colecistoquinéticos); acudió a consulta en Urgencias Adulto con intenso dolor en el epigastrio acompañado de náusea que llegaba a la emesis de contenido gastroalimentario y luego biliar, acompañado de diaforesis y palidez. Se decidió su ingreso con diagnóstico de enfermedad ácido péptica. Se inició un protocolo de estudios con solicitud de biometría hemática (Tabla 1), química sanguínea (Figura 1 y Tabla 2), pruebas funcionales hepáticas (no se obtuvieron de ingreso por suero lipémico) (Tabla 2). La exploración abdominal reveló dolor intenso a la palpación en el epigastrio, útero grávido a $17 \mathrm{~cm}$ de la sínfisis del pubis, peristalsis disminuida. Se realizó un ultrasonido 
abdominal (no contamos con la imagen, sólo el reporte escrito), con los siguientes hallazgos: páncreas de aspecto adenomatoso, lodo biliar en la vesícula, colédoco y porta no valorables (Figura 1). Ultrasonido obstétrico: producto único de 19.5 SDG; frecuencia cardiaca fetal de 156/min; índice de líquido amniótico apreciativamente normal, placenta fúndica anterior. Tras ello se dio el diagnóstico de pancreatitis aguda secundaria a dislipidemia; se solicitó la valoración por la unidad de cuidados intensivos y se aceptó.

\section{Curso y evolución}

Se ingresó a las ocho horas en terapia intensiva, donde persistió con acidosis metabólica e hiperlipidemia; se le brindó tratamiento médico a base de ayuno, hidratación parenteral, sonda nasogástrica, bloqueador $\mathrm{H} 2$, analgesia a base de opioide tipo tramadol; no hubo decre-

Tabla 1. Datos biometría hemática.

\begin{tabular}{ccrcccr}
\hline & TA & FC & FR & SatO $_{2}$ & Temperatura & PVC \\
\hline Ingreso & $120 / 80$ & 85 & 16 & $92 \%$ & 36 & \\
24 h & $110 / 50$ & 115 & 22 & $94 \%$ & 36.5 & 3 \\
48 h & $120 / 60$ & 120 & 19 & $99 \%$ & 36 & 7 \\
5 día & $130 / 60$ & 103 & 20 & $93 \%$ & 36.7 & 14 \\
8 día & $125 / 60$ & 104 & 20 & $94 \%$ & 36.5 & 10 \\
\hline
\end{tabular}

Tabla 2: Datos de laboratorio para diagnóstico de pancreatitis grave.

\begin{tabular}{ccc}
\hline & Hematocrito & Leucocitos \\
\hline Día 1 & 41.6 Pérdida $>10 \%$ & $15.6>16,000 / \mathrm{mm}^{3}$ \\
Día 2 & 33.5 & 8.78 \\
Día 5 & 30.1 & 7.07 \\
Día 8 & 32.6 & 5.68 \\
\hline
\end{tabular}

Tabla 3: Criterios de ingreso y pronóstico para pancreatitis grave.

\begin{tabular}{ccccccc}
\hline Ranson & & APACHE & & BISAP & ATLANTA & Balthazar \\
\hline $24 \mathrm{~h}$ & 3 & Al ingreso & 20 & 1 & $\begin{array}{c}\text { Pancreatitis aguda } \\
\text { moderadamente } \\
\text { severa }\end{array}$ & $\mathrm{B}$ \\
$48 \mathrm{~h}$ & 3 & $48 \mathrm{~h}$ & 7 & & & \\
\hline
\end{tabular}

Tabla 4: Datos de Laboratorio de Pancreatitis secundaria a dislipidemia mixta.

\begin{tabular}{lcccccc}
\hline & Colesterol & Triglicéridos & Amilasa & Lipasa & DHL & Glucosa \\
\hline Día 1 & $\begin{array}{c}705<200 \\
\mathrm{mg} / \mathrm{dL}\end{array}$ & $\begin{array}{c}2,840<150 \\
\mathrm{mg} / \mathrm{dL}\end{array}$ & 1,117 & 1,200 & $\begin{array}{c}289>350 \\
\mathrm{U} / \mathrm{L}\end{array}$ & 256 \\
Día 2 & 507 & $\begin{array}{c}1,711<200 \\
\mathrm{mg} / \mathrm{dL}\end{array}$ & 1,735 & 443 & 752 & $\begin{array}{c}240>200 \\
\mathrm{mg} / \mathrm{dL}\end{array}$ \\
& & & & & 120 \\
Día 3 & 350 & 705 & 262 & 127 & 725 & \\
Día 4 & 359 & 659 & & & & 150 \\
Día 5 & & & 152 & 124 & & 257 \\
Día 6 & 403 & 676 & 89 & 137 & & 193 \\
Día 7 & 301 & 502 & & & 19 & 187 \\
Día 8 & 477 & 256 & & & & \\
\hline
\end{tabular}

mento de los triglicéridos y el colesterol, por lo que se decidió programar plasmaféresis dentro de las primeras 48 horas de estancia (Tabla 3). Sin embargo, presentó al tercer día reducción de los valores; con apoyo de la valoración del Servicio de Hematología se difirió dicho procedimiento (Tabla 4).

Se mantuvo en ayuno, ya que persistía con dolor abdominal; inició la nutrición parenteral sin lípidos; posteriormente, reinició tolerancia a la vía oral, con buena aceptación, así como ausencia de dolor y buena tolerancia a la dieta, sin compromiso obstétrico. Se egresó a hospitalización y 48 horas después se egresó a domicilio por evolución favorable.

Se realizaron estudios de seguimiento familiar, la cual estaba compuesta de siete integrantes (incluida nuestra paciente) de 16 a 25 años de edad, cuatro mu-

Tabla 5: Valores de laboratorio de familiares directos.

\begin{tabular}{cccccr}
\hline & Edad & Glucosa & Colesterol & Triglicéridos & DHL \\
\hline Padre & 50 & & 250 & 289 & \\
Madre & 45 & 254 & 134 & 170 & 27 \\
Hermana & 25 & 315 & 284 & 2,229 & 295 \\
Hermano & 23 & 93 & 238 & 363 & \\
Hermana & 20 & 396 & 458 & 3,697 & \\
Hermano & 18 & 120 & 161 & 173 & \\
\hline
\end{tabular}

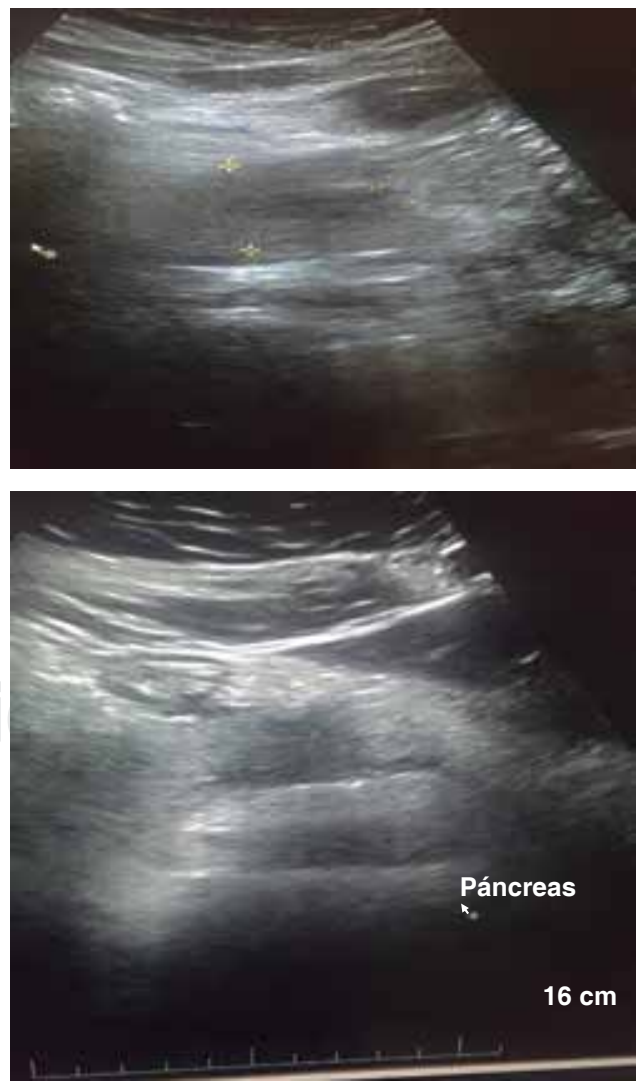

Figura 1: Páncreas aspecto adenomatoso. 
jeres y tres hombres; la madre de 45 años tenía como antecedente de importancia diabetes mellitus tipo 2 e insuficiencia renal terminal con manejo sustitutivo hemodiálisis, por lo que concluimos que la familia es portadora de dislipidemia mixta familiar (Tabla 5).

\section{DISCUSIÓN}

La pancreatitis aguda ocurre en 0.03 a $0.09 \%$ de los embarazos. La enfermedad de cálculos biliares es responsable de aproximadamente el $70 \%$ de estos casos. En el presente caso se concluyó que la causa fue metabólica, secundaria a dislipidemia; ${ }^{4}$ tenía múltiples factores que la predisponían a desarrollar este evento: primero, la predisposición familiar; el evento previo de pancreatitis; la diabetes mellitus; el embarazo y alto riesgo de recurrencia. ${ }^{5}$ Este tipo de padecimiento en la mujer embarazada debe ser manejado por un equipo multidisciplinario para mejorar la evolución, pronóstico y la supervivencia del binomio. ${ }^{6}$

\section{BIBLIOGRAFÍA}

1. Pitchumoni CS, Yegneswaran B. Acute pancreatitis in pregnancy. World J Gastroenterol. 2009;15(45):5641-5646.
2. Ducarme G, Maire F, Chatel P, Luton D, Hammel P. Acute pancreatitis during pregnancy: a review. J Perinatol. 2014;34(2): 87-94.

3. Tang SJ, Rodriguez-Frias E, Singh S, Mayo MJ, Jazrawi SF, Sreenarasimhaiah $\mathrm{J}$, et al. Acute pancreatitis during pregnancy. Clin Gastroenterol Hepatol. 2010;8(1):85-90.

4. Aguilar-Salinas CA, Huertas A, Tusié MT, Gómez-Pérez FJ, Rull JA. Hiperlipidemia familiar combinada: caracterización en población mexicana. Rev Endocrinol Nutr. 2002;10(2):58-62.

5. Briones-Garduño JC, García-Ochoa ED, Zavala-Castillo JC, Campos-Castillo C, Sandoval-Ayala OI, Briones-Vega CG, et al. Pancreatitis y embarazo. Rev Asoc Mex Med Crit y Ter Int. 2014;28(3):159-163.

6. Ntzeros K, Fragiadakis I, Stamatakos M. Acute pancreatitis in pregnancy-Up to Date. Open J Obstet Gynecol. 2014;4:81-89.

Patrocinios y conflicto de intereses: Los autores declaramos no haber recibido patrocinio para el presente trabajo ni tener conflicto de intereses.

Correspondencia:

Dra. María Guadalupe Hernández Landeros Hospital Regional

de Alta Especialidad del Bajío

Blvd. Milenio Núm. 130,

Col. San Carlos la Roncha,

37600, León, Gto.

Tel: 044 (477) 5776822

E-mail: lupitahdez31@hotmail.com 